\title{
Ansiedad y nivel de conocimiento en cuidados paliativos en estudiantes de Guerrero
}

\author{
Anxiety and level of knowledge in palliative care in students from Guerrero
}

\section{Ansiedade e nível de conhecimento em cuidados paliativos em alunos de Guerrero}

\author{
Elvia Peña-Marcial ${ }^{1}$ \\ Jorge Sait Díaz-Marcelo ${ }^{2}$ \\ Leticia Reyna-Avila ${ }^{3}$ \\ Rodrigo Pérez-Cabañas ${ }^{4}$ \\ Lorena Inés Bernal-Mendoza ${ }^{5}$
}

\section{Resumen}

Objetivo: Identificar el nivel de conocimiento en cuidados paliativos y ansiedad ante la muerte en estudiantes de las escuelas de enfermería, de la Universidad Autónoma de Guerrero. Materiales y métodos: Estudio mixto, comparativo y fenomenológico mediante la narrativa testimonial. La selección se realizó con un muestreo aleatorio simple con reemplazo, teniendo como referencia el listado de estudiantes legalmente inscritos en cuarto año de licenciatura en Enfermería de tres escuelas. La población total fue de 380 encuestados de ambos sexos para el estudio cuantitativo. También, se utilizó un instrumento autoaplicado adaptado y validado por los investigadores, integrado por tres apartados: datos sociodemográficos, nivel de conocimiento de cuidados paliativos (Palliative Care Knowledge Test (-PCKT) y el miedo a la muerte (Escala de Miedo a la Muerte de Collett-Lester). Para el estudio cualitativo se incluyeron seis participantes de cada escuela, dando un total de 18 seleccionados al azar, a quienes que se les realizó una entrevista a profundidad ( 25 a 40 minutos) con ocho preguntas abiertas y grabadas; además, se efectuaron notas en un diario de campo para identificar el estado emocional de los participantes. Resultados: El $78.7 \%$ de los estudiantes tiene un nivel de conocimientos en cuidados paliativos malo. Según la escala de ansiedad y miedo a la muerte de Collett-Lester, el $66.3 \%$ percibe un nivel muy ansioso/a. Por otro lado, de los cuatro panoramas de muerte se encontró que: el 55.3\% le tenía miedo a la propia muerte y era moderadamente ansioso/a; al propio proceso de morir $57.1 \%$ muy ansioso/a; a la muerte de otros $75.5 \%$ muy ansioso/a y al proceso de morir de otros $60 \%$ muy ansioso/a. Ante el proceso de la muerte describieron sentimientos de: tristeza, ansiedad, vacío profundo, desesperación, temor, frustración, enojo, actitud de autocontrol y aceptación. Las intervenciones que emplearían con el paciente hospitalizado en etapa terminal y con los familiares serían técnicas y procedimientos para prevenir otras patologías y el alivio del dolor. Conclusión: Los estudiantes tienen un nivel de conocimiento malo en cuidados paliativos, porque el plan de estudios no integra ninguna unidad de aprendizaje específica para este cuidado. El nivel de ansiedad ante la muerte tuvo una representación muy alta y los estudiantes se perciben muy ansiosos/as ante los procesos de la muerte.

Palabras Clave: Temor; Tanatología; Cuidado terminal; Enfermería.

\section{Abstract}

Objective: To identify the level of knowledge in palliative care and anxiety before the death of the students of the nursing schools of the Autonomous University of Guerrero. Materials and methods: Mixed, comparative and phenomenological study through testimonial narrative. The selection was made with a simple random sampling with replacement taking as a reference

\section{Autor de correspondencia*}

1* Maestra en Salud Pública. Enfermera operativa de la Clínica Hospital ISSSTE de Chilpancingo; Guerrero. Docente, Universidad Autónoma de Guerrero. Chilpancingo, México. Correo: elviapema@hotmail.com (1D) 0000-0003$1232-6000$

${ }^{2}$ Licenciatura en Enfermería. Enfermero General - Operativo del Hospital General Raymundo Abarca Alarcón de Chilpancingo; Guerrero. Correo: jorge diaz9617@, hotmail.com (iD) 0000-0001-

${ }^{3}$ Maestría en Enfermería. Docente Universidad Autónoma de Guerrero. Chilpancingo, México. Correo: crysle81@ hotmail.com (1) 0000-0001-9862-4062

${ }^{4}$ Maestría en estadística aplicada. Docente, Universidad Autónoma de Guerrero. Correo: 05575@uagro.mx (ib00000002-3417-2308

5 Maestra en Salud Pública. Docente Universidad Autónoma de Guerrero. Chilpancingo, México. Correo: lorenaines@hotmail.com (1) 0000-0002$\underline{1799-9522}$

Recibido: 22 julio 2021

Aprobado: 28 mpviembre 2021

Para citar este artículo

Peña-Marcial E, Diaz-Marcelo JS, Reyna-Avila L, Peérez-Cabañas R, Bernal-Mendoza LI. Ansiedad y nivel de conocimiento en cuidados paliativos en estudiantes de Guerrero. Rev. cienc. cuidad. 2022; 19(1):57-70. https://doi. org/10.22463/17949831.3114

(C) Universidad Francisco de Paula Santander. Este es un artículo bajo la licencia CC-BY-NC-ND 


\section{Scientific Journal of Nursing}

the list of students legally enrolled in the fourth year of the Bachelor's Degree in Nursing from three schools. The total population was 380 respondents of both sexes for the quantitative study, a self-applied instrument adapted and validated by the researchers was used, consisting of three sections, sociodemographic data, level of knowledge of palliative care (PCKT) and fear of death (Collett-Lester Fear of Death Scale). Para el estudio cualitativo se incluyeron seis participantes de cada escuela teniendo un total de 18 seleccionados al azar a los que se les realizó una entrevista a profundidad (25 a 40 minutos) con ocho preguntas abiertas, grabadas y notas en un diario de campopara identificar el estado emocional de los participantes. Results: $78.7 \%$ of the students have a poor level of knowledge in palliative care. According to the Collett-Lester scale of anxiety and fear of death, $66.3 \%$ perceive a very anxious level, of the four death scenarios it was found that, at death itself, $55.3 \%$ moderately anxious; the very process of dying $57.1 \%$ very anxious; to the death of another $75.5 \%$ very anxious and to the dying process of another $60 \%$ very anxious. Faced with the death process, they described feelings of sadness, anxiety, deep emptiness, despair, fear, frustration, anger, an attitude of self-control, acceptance. The interventions that they would use, in the terminal hospitalized patient and with the relatives, would be techniques and procedures to prevent other pathologies and relieve pain. Conclusion:students have a poor level of knowledge in palliative care, because the curriculum does not include any specific learning unit for this care. The level of anxiety before death had a very high representation, the students perceived themselves as very anxious before the death processes.

Keywords: Fear; Thanatology; Terminal Care; Nursing.

\section{Resumo}

Objetivo: Identificar o conhecimento em cuidados paliativos e ansiedade perante a morte em alunos de escolas de enfermagem da Universidade Autônoma de Guerrero. Materiais e métodos: Estudo misto, comparativo e fenomenológico usando narrativa testemunhal. A seleção realizou-se de forma aleatória simples com substituições, usando como referência o listado de alunos matriculados no quarto ano da licenciatura em enfermagem de três escolas. A amostra do estudo quantitativo foi de 380 sujeitos de ambos os sexos. Empregou-se um instrumento auto aplicado, adaptado e validado pelos pesquisadores, integrado por três componentes: informação demográfica; nível de conhecimento em cuidados paliativos (Palliative Knowledge Test-PCKT ) e medo da morte (escala de medo da morte de Collet-Lester). Para a pesquisa qualitativa incluíram-se 6 alunos de cada escola, completando 18 participantes escolhidos ao azar e aplicou-se uma entrevista a profundidade com 8 perguntas abertas. Além disso, empregaram-se anotações do diário de campo para identificar o estado emocional dos participantes. Resultados: O 78,7\% dos alunos têm maus conhecimentos sobre cuidados paliativos. Segundo a escala de Collet-Lester, o $66,3 \%$ dos alunos se percebe muito ansioso. Por outro lado, dos quatro panoramas da morte encontrou-se que $55,3 \%$ têm medo da própria morte e era moderadamente ansioso/a; ao próprio processo de morrer de outros $57,1 \%$ muito ansioso/a; a morte dos outros $75,5 \%$ muito ansioso/a e ao processo de morrer outros $60 \%$ muito ansioso/a. Perante o processo da morte descreveram sentimentos de tristeza, ansiedade, vazio profundo, desesperação, medo, frustração, raiva, atitude de autocontrole e aceitação. As intervenções que teriam com o paciente em etapa terminal e com os seus familiares seriam técnicas e procedimentos par prever outras patologias e o alivio da dor. Conclusão: Os alunos têm maus conhecimentos de cuidados paliativos, porque o plano de estudos não contém nenhuma unidade de aprendizagem específica para esse tema. O nível de ansiedade perante a morte e os processos da mesma, tiveram representações elevadas.

Palavras-chave: Medo; Tanatologia; Enfermagem; Assistência terminal. 


\section{Introducción}

Es muy importante la preparación dentro de las escuelas de licenciados en enfermería, para que así puedan brindar cuidados paliativos de forma integral, ya que son quienes constituyen la mayor fuerza de trabajo del sector salud, público y privado, y en su mayoría no reciben una preparación acorde con esta área $(1,2)$.

El conocimiento tiene que ver con hechos o información que adquiriere una persona a través de la experiencia o la educación y la comprensión teórica o práctica de un asunto referente a la realidad; factores que implican una dualidad de realidades: de un lado, el sujeto cognoscente y, del otro, el objeto conocido, que es poseído en cierta manera, por el sujeto cognoscente $(3,4)$.

La Organización Mundial de la Salud (OMS) define los cuidados paliativos $(\mathrm{CP})$ como "el enfoque que mejora la calidad de vida de pacientes y familias que se enfrentan a los problemas asociados con enfermedades amenazantes para la vida, a través de la prevención y el alivio del sufrimiento, por medio de la identificación temprana y la impecable evaluación y tratamiento del dolor y otros problemas físicos, psicosociales y espirituales". Igualmente, considera que el equipo socio sanitario debe aproximarse a los enfermos y a sus familiares con el objetivo de responder a sus necesidades $(5,6)$.

Hay que mencionar, además que la ansiedad es una emoción que surge ante situaciones ambiguas, en las que se anticipa una posible amenaza, y que prepara al individuo para actuar ante ellas mediante una serie de reacciones cognitivas, fisiológicas y conductuales. Se trata de un sentimiento subjetivo que implica la presencia de aprensión, tensión, inquietud, temor indefinido e inseguridad ante un peligro no aclarado (7).

Por otro lado, la muerte, al igual que el nacimiento, es un fenómeno natural inherente a la condición humana. Ambos incluyen características sociales culturales y biológicas, además de las facetas psicológicas, emocionales y espirituales. A lo largo de la vida del ser humano estos dos eventos son quizá los que producen mayor emoción, aunque la respuesta emocional y la consideración social frente a cada acontecimiento son muy diferentes (8). El cómo muere la gente, permanece en la mente de los que continúan viviendo.

Conviene subrayar que los cuidados paliativos mejoran la calidad de vida de los pacientes y sus allegados cuando afrontan problemas de orden físico, psicosocial o espiritual inherentes a una enfermedad potencialmente mortal. Los cuidados paliativos se han condicionado a los hospitales oncológicos, pero su aplicación es universal. Al respecto la OMS indica que hay 40 millones de personas que necesitan cuidados paliativos, determinando que el $78 \%$ corresponde a países con ingreso bajo e ingreso mediano; sin embargo, a nivel mundial, tan solo un $14 \%$ de ese porcentaje los reciben $(9,10)$. Además, se agrega que todas las personas tienen derecho a una asistencia de calidad científica y humana, por lo que recibir una adecuada atención al final de la vida no debe ser considerado un privilegio sino un auténtico derecho (11).

Se sabe que los cuidados paliativos a pacientes terminales desde la percepción de los profesionales de la enfermería está dirigido a mejorar la calidad de vida del paciente frente al dolor y a mitigar el sufrimiento del familiar, a través de la comunicación y la valorización de la vida. Por tanto es fundamental la capacitación para el cuidado del paciente, así como la importancia de la proximidad con los pacientes en el final de la vida, siendo relevante su actuación para mejorar su calidad de vida y mitigar el sufrimiento (12).

Como lo menciona Jean Watson en su teoría del cuidado transpersonal, el ideal moral de la enfermería es la protección, mejora y preservación de la dignidad humana. Es por eso que el cuidado humano involucra: valores, voluntad y un compromiso para cuidar, conocimiento, acciones de cuidado y consecuencias. Al considerar el cuidado como intersubjetivo, responde a procesos de salud y enfermedad, interacción persona y medio ambiente, conocimientos de los procesos de cuidado de la enfermería, autoconocimiento, conocimiento del poder de sí mismo y limitaciones en la relación de cuidado. Watson conceptualiza el cuidado como un proceso interpersonal entre dos personas, con dimensión transpersonal (enfermera paciente). De la misma forma, según esta autora, el cuidado inicia cuando la/ el enfermera/o entra en el campo fenomenológico del paciente (Marco de referencia de la persona, realidad subjetiva compuesta en su totalidad por la experiencia humana) y responde a la condición de ser del paciente (espíritu, alma) de la misma forma en que el paciente expone sus sentimientos subjetivos (13).

En cuanto al significado otorgado por las enfermeras al cuidado humanizado se constata que aunque tienen 
conocimientos y conciencia de su importancia, encuentran dificultades a la hora de implementarlo en la práctica; es decir, que la satisfacción de este tipo de necesidades adopta un papel secundario respecto a las intervenciones técnicas y tecnológicas, formando parte de lo que se conoce como "cuidado invisible", lo que dificulta que la sociedad visualice la aportación específica de las enfermeras (14).

Es necesario recalcar, por una parte, sobre la importancía del bienestar propio en todas sus dimensiones, para poder ofrecer una ayuda efectiva a quien sufre; $y$, por otra, la necesidad de trabajar las propias dimensiones que tienen que ver con lo espiritual, lo social y lo emocional (15).

Definitivamente, hacer frente a los problemas reales, es responsabilidad de las escuelas de enfermería antes de enviar a los estudiantes a realizar prácticas clínicas; no obstante, el carecer de formación adecuada para enfrentar los procesos de la muerte, marcan a la persona y al estudiante de manera permanente $(16,17)$.

En el día a día sobre la formación de nuevos recursos humanos de la enfermería, las escuelas del país y del mundo deben tener el firme compromiso y la obligación de perfeccionar aquellos factores que coadyuven en la mejora constante, tales como: maestros preparados, laboratorios equipados y funcionales, excelentes planes de estudio y campos clínicos, de tal forma que puedan darles a sus egresados una amplia capacidad resolutiva, en todo aquellos cuidados que la población necesita.

Por todo lo anterior, es importante profundizar en la formación de los estudiantes de enfermería en los cuidados paliativos, considerando los criterios de calidad en la atención (11).

Por consiguiente, las enfermeras deben estar bien preparadas en cuidados paliativos, para que brinden la atención existencial y espiritual a los pacientes, facilitando la relación enfermera-paciente; además, hay que utilizar estrategias que mejoren la educación en esta área (18).

\section{Objetivos}

\section{Objetivo general}

Identificar el nivel de conocimiento en cuidados palia- tivos y ansiedad ante la muerte de los estudiantes de las escuelas de enfermería, de la Universidad Autónoma de Guerrero, México.

\section{Objetivos específicos}

- Identificar el nivel de conocimiento sobre cuidados paliativos que tienen los estudiantes.

- Identificar el nivel de ansiedad ante la muerte que tiene los estudiantes.

- Identificar el estado emocional de los estudiantes ante procesos de duelo o muerte de diferentes panoramas.

- Conocer si el estado emocional influye en los cuidados paliativos otorgados por los estudiantes.

\section{Materiales y métodos}

Se realizó un estudio mixto, comparativo y fenomenológico mediante la narrativa testimonial en alumnos de las escuelas de enfermería de la Universidad Autónoma de Guerrero (UAGro), en Chilpancingo de los Bravo, Coyuca de Catalán y Acapulco de Juárez, entre abril y mayo de 2019.

La muestra se calculó con el programa public domain statistical software for epidemiology (Epi Info) versión 7 con un nivel de confianza del 95\% y un 5\% de error. La selección se realizó mediante un muestreo aleatorio simple con reemplazo, teniendo como referencia el listado de alumnos legalmente inscritos; al respecto no se manipularon las variables de estudio.

La investigación se efectuó con estudiantes de cuarto año de licenciatura, con una población total de 380 de ambos sexos (146 pertenecen a la Escuela de Enfermería No. 1, 188 a la Escuela de Enfermería No. 2 y 46 a la Escuela de Enfermería No. 5). Para el estudio cualitativo se incluyeron seis participantes de cada escuela obteniendo un total de 18, seleccionados al azar. Los criterios de selección estuvieronsujetos a: que estudiaran en las Escuelas de Enfermería No. 1, 2 y 5; que se encontraran inscritos legalmente; y, que dieran su consentimiento para participar.

Se efectuó una prueba piloto aplicando 10 encuestas a un grupo de alumnos de otros grados académicos de la Escuela Superior de Enfermería No. 1 en marzo de 2019. Con esta prueba piloto se calculó el nivel de con- 


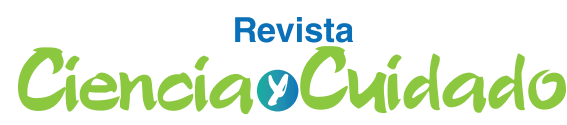

Scientific Journal of Nursing

fiabilidad mediante el programa estadístico SPSS v21. Se empleó un instrumento autoaplicado adaptado y validado por los investigadores, integrado por tres apartados: el primero incluye los datos sociodemográficos con 8 Ítems; el segundo mide el nivel de conocimiento de cuidados paleativos, conformado por 20 ítems, agrupado en cinco dimensiones (filosofía, dolor, disnea, problemas psiquiátricos y problemas gastrointestinales) teniendo como referente el cuestionario Palliative Care Knowledge Test (PCKT) (19), con un nivel de confiabilidad (alpha de Crombach) de 0.739. Por otra parte, para el análisis de las dimensiones en las que se mide el conocimiento, se hicieron cuatro cortes con intervalos de: 0 a 10 nivel malo de conocimiento, de 11 a 15 regular, de 16 a 18 bueno y 19 a 20 excelente. El tercer apartado mide el miedo a la muerte, aplicando la Escala del Miedo a la Muerte de Collett-Lester, que consta de 28 preguntas compuestas por 4 dimensiones que relacionan 7 panoramas distintos de una misma circunstancia, con las siguientes opciones de respuesta: Nada, Moderado y Mucho. De igual forma se asignó el valor de 1 "Nada Ansioso/a", 2 "Moderadamente Ansioso/a" y 3 "Muy Ansioso/a". Posteriormente se efectuó la sumatoria y se dividió en tres cortes: de 1 a 7 "Nada Ansioso/a", de 8 a 14 "Moderadamente Ansioso/a" y de 15 a 21 "Muy Ansioso/a", (20) con un nivel de confiabilidad (alpha de Crombach) de 0.849.

Es importante señalar que para la investigación cualitativa retomando la idea de los nuevos retos de la investigación, se aplicó un cuestionario a profundidad con ocho preguntas abiertas, además se grabaron; también se efectuaron notas en un diario de campo. El análisis y transcripción permitió identificar el estado emocional de los participantes, teniendo en cuenta que cada entrevista se estudió de forma unitaria desde el principio para identificar situaciones particulares y posteriormente organizar las diferentes respuestas en un solo contenido, respetando las ideas centrales de los participantes (21).

Al mismo tiempo se realizó la solicitud a los directivos de las Escuelas seleccionadas, quienes dieron la autor-
Ansiedad y nivel de conocimiento en cuidados palia-

tivos en estudiantes de Guerrero ización. El procedimiento para levantar la información fue mediante la autoaplicación de la encuesta a los 380 estudiantes elegidos en la muestra, previa firma del consentimiento informado, cumpliendo con el Reglamento de la Ley General de Salud en materia de Investigación (22).

Para el procesamiento de la información se utilizó el programa IBM SSPS Statistics 21. Inicialmente se hizo un análisis estadístico descriptivo y posteriormente un análisis univariado y bivariado; seguidamente se efectuó un análisis de asociación usando la tau-b y tau-c de kendall, así como la búsqueda de dependencia de variables, a través de la Chi-cuadrada de Pearson; se consideró significativo un valor de $\mathrm{p}<0.05, \mathrm{IC}=95 \%$. En las variables ordinales se usó el coeficiente tau b y tau c de Kendall, estos asumen valores entre -1 y 1; de acuerdo con el nivel de significancia del coeficiente, se concluye si existe dependencia significativa entre el nivel de conocimiento, la ansiedad y miedo a la muerte. Para el análisis de datos cualitativos se transcribieron las entrevistas grabadas y los relatos del diario de campo, los cuales mostraron las vivencias y permtieron la reflexión ante la situación concreta. Se codificaron las transcripciones; después se integraron los códigos coincidentes en subtemas, dando origen a dos temas (1. Sentimientos ante la muerte y 2. Cuidados paliativos); posteriormente los hallazgos fueron teorizados y fundamentados con las entrevistas

\section{Resultados}

En el presente estudio se incluyeron 380 estudiantes, el mayor porcentaje corresponde a la Escuela de Enfermería No. 2, predominio el sexo femenino, la edad promedio fue de $22 \pm 2.332$ años, el mayor porcentaje de los encuestados es soltero y profesa la religión católica (ver tabla 1). 
Scientific Journal of Nursing

Tabla 1: Caracterización sociodemográfica de los estudiantes

\begin{tabular}{llll}
\hline & Variable & \multicolumn{1}{c}{ F } & \% \\
\hline Edad & 20-24 años & 338 & 88.9 \\
25-29 años & 36 & 9.5 \\
Sexo & Femenino & 30 anos & 1.6 \\
Estado Civil & Masculino & 72 & 81.1 \\
& Soltero/a & 329 & 86.6 \\
& Casado/a & 29 & 7.6 \\
Religión & Unión Libre & 17 & 4.5 \\
& Divorciado/a & 5 & 1.3 \\
& Católica & 309 & 81.3 \\
& Cristiana & 30 & 7.9 \\
& Testigo de Jehová & 9 & 2.4 \\
& Evangelista & 2 & 0.5 \\
Escuela & Otras & 30 & 7.9 \\
& Enfermería No. 1 & 146 & 38.4 \\
& Enfermería No. 2 & 188 & 49.5 \\
& Enfermería No. 5 & 46 & 12.1 \\
\hline
\end{tabular}

Fuente: Encuesta a estudiantes de enfermería de la UAGro Abril-Mayo 2019

Con respecto a la la prevalencia de la escala sobre ansiedad y miedo a la muerte de Collett-Lester, en general se encontró que el $66.3 \%$ perciben un nivel muy ansioso/a. En el análisis de cada uno de los cuatro panoramas de muerte se encontró que: $55.3 \%$ está moderadamente ansioso/a, en relación a la propia muerte; $57.1 \%$ tiene mucho miedo con respecto al propio proceso de morir; $75.5 \%$ tiene mucho miedo en relación a la muerte de otros; y, 60\% mucho en relación al proceso de morir de otros.
Desde otro punto de vista, respecto al nivel de conocimiento en cuidados paliativos que tienen los estudiantes de enfermería de la UAGro se encontró que: el $78.7 \%$ tiene un nivel malo con un puntaje de entre 0 a 10. En la distribución por escuela se observó que: la escuela de Enfermería No. 1 obtuvo un 80.1\% malo, Enfermería No. 2 un $75 \%$ malo, y Enfermería No. 5 un $89.1 \%$ malo (ver tabla 2 ) 
Scientific Journal of Nursing

Tabla 2: Nivel de conocimiento en cuidados paliativos y ansiedad ante la muerte en estudiantes

\begin{tabular}{|c|c|c|c|}
\hline Variable & & $\mathrm{F}$ & $\%$ \\
\hline \multirow{2}{*}{$\begin{array}{c}\text { ¿Sabes cuáles son los cuidados palia- } \\
\text { tivos? }\end{array}$} & $\mathrm{Si}$ & 374 & 98.4 \\
\hline & No & 6 & 1.6 \\
\hline \multirow[t]{4}{*}{ Percepción de nivel de conocimiento } & Malo & 16 & 4.2 \\
\hline & Regular & 253 & 66.6 \\
\hline & Bueno & 106 & 27.9 \\
\hline & Excelente & 5 & 1.3 \\
\hline \multirow{4}{*}{$\begin{array}{l}\text { Nivel de conocimiento en cuidados pa- } \\
\text { liativos }\end{array}$} & Malo & 299 & 98.4 \\
\hline & Regular & 79 & 20.8 \\
\hline & Bueno & 2 & 0.5 \\
\hline & Excelente & 0 & 0 \\
\hline \multirow{3}{*}{$\begin{array}{l}\text { Grado de preocupación o ansiedad } \\
\text { ante la muerte en relación a la propia } \\
\text { muerte }\end{array}$} & Nada & 16 & 4.2 \\
\hline & Moderado & 210 & 55.3 \\
\hline & Mucho & 154 & 40.5 \\
\hline \multirow{3}{*}{$\begin{array}{l}\text { Grado de preocupación o ansiedad ante } \\
\text { la muerte en relación al propio proceso } \\
\text { de morir }\end{array}$} & Nada & 8 & 2.1 \\
\hline & Moderado & 155 & 40.8 \\
\hline & Mucho & 217 & 57.1 \\
\hline \multirow{3}{*}{$\begin{array}{l}\text { Grado de preocupación o ansiedad } \\
\text { ante la muerte en relación a la muerte } \\
\text { de otros }\end{array}$} & Nada & 5 & 1.3 \\
\hline & Moderado & 88 & 23.2 \\
\hline & Mucho & 287 & 75.5 \\
\hline \multirow{3}{*}{$\begin{array}{l}\text { Grado de preocupación o ansiedad } \\
\text { ante la muerte en relación al proceso } \\
\text { de morir de otros }\end{array}$} & Nada & 11 & 2.9 \\
\hline & Moderado & 141 & 37.1 \\
\hline & Mucho & 228 & 60 \\
\hline
\end{tabular}

Fuente: Encuesta a estudiantes de enfermería de la UAGro Abril-Mayo 2019

Por otro lado, al relacionar la variable sobre el nivel de conocimiento no se encontró una dependencia con la propia muerte, pero si una asociación y una correlación positiva; al relacionarse con el propio proceso de morir de igual forma no se encontró una dependencia; no obstante, si se hace una asociación y una correlación positiva con la variable muerte de otros, se encontró una dependencia muy significativa, asociada a una correlación positiva, pero al relacionarla con la variable proceso de morir de otros, se encontró una dependencia significativa en lo que respecta a la asociación, con una correlación positiva (ver tabla 3 ). 
Scientific Journal of Nursing

Tabla 3. Asociación del nivel de conocimiento de cuidados paliativos y la ansiedad ante la muerte en estudiantes

\begin{tabular}{cc} 
Variables & Valores \\
\hline Nivel de conocimiento en cuidados paliativos y nivel de ansiedad & Chi- $2=0.703$ \\
ante la propia muerte & Tau-c $=0.609$ \\
& R de Pearson $=0.691$ \\
Chi- $2=0.977$ \\
Tau-c $=0.8$ \\
ante el propio proceso de muerte & R de Pearson $=0.763$ \\
& \\
Chivel de conocimiento en cuidados paliativos y nivel de ansiedad & Tau-c $=0.000$ \\
ante la muerte de otros & R de Pearson $=0.355$ \\
& \\
Chi-2 $=0.002$ \\
Tivel de conocimiento en cuidados paliativos y nivel de ansiedad \\
ante el proceso de morir de otros & R de Pearson $=0.601$ \\
& \\
\hline
\end{tabular}

Fuente: Encuesta a estudiantes de enfermería de la UAGro Abril-Mayo 2019

A continuación se describen los resultados de las entreA continuación se describen los resultados de las entrevistas a profundidad, realizadas a los 18 estudiantes de las tres escuelas de enfermería. Se presentan dos categorías resultantes del análisis cualitativo que describen el nivel de conocimiento y ansiedad ante la muerte en los estudiantes.

\section{Sentimientos ante la muerte}

Los estudiantes mencionan que si les avisaran del fallecimiento de algún familiar tendrían una reacción de sorpresa e impresión al recibir una noticia de tal magnitud $_{(01,02,04,06,07,09,11,13)}$; otros que sentirían tristeza y lo demostrarían mediante lágrimas (llanto) ${ }_{(01,03,04,06,08,}$ $09,10,12,14,15,17,18$, Igualmente, tendrían un sentimiento de ansiedad, vacio profundo y desesperación ${ }_{(02,08,14)}$, así como sentimiento de temor, frustración, enojo e ira ${ }_{11}$, ${ }_{14,18 .}$. Por su parte algunos consideran que experimentarían una actitud de autocontrol, aceptación y tratarían de asimilarla ${ }_{(02,05,13,16)}$.

"La verdad no sé cómo respondería mi cuerpo y mi mente ante esa situación"

"A través de mis gestos, a través de quizás mi estado de ánimo"

"si estuviera enfermo como que sería de esperarse" ${ }_{(07)}$.
Por otro lado, los entrevistados mencionan las reacciones y lo que le dirían a sus familiares si estuvieran durante el proceso de muerte de algún familiar cercano, que pueden ser de diferentes formas; es decir, algunos estudiantes coinciden que tendrían el mismo sentimiento ${ }_{(02,05,06,07,10)}$ que en la situación de una persona que no sea familiar; no obstante para algunos estar durante el proceso de muerte de un familiar les da tranquilidad y consuelo el haber estado durante este fase, así como de poder apoyar a su paciente enfermo y a su familia $(02,03,04,06,10,11,12,13,15)$. De igual forma, los participantes establecen la diferencia cuando el familiar va a fallecer o falleció, pero estaba pasando por un proceso de enfermedad que le provocara molestias y no le dejara desarrollar correcta y efectivamente su vida, por tanto, es mejor el morir que estar sufriendo ${ }_{(13,15,17)}$ y se debe aceptar para que haya resignación $(01,08,14,17,18)$, ya que la muerte es parte del proceso de vivir y algún día tiene que pasar ${ }_{(13,16)}$.

"No es algo que aceptamos al principio, esto lleva su tiempo para llegar a la resignación" ${ }_{(18)}$.

"Tenemos que morir algún día"

"No tenemos las palabras adecuadas para expresar o consolar a la familia" (07).

"El hecho de que él llegara a fallecer sería como un descanso que él tendría del padecimiento que él tuviera" (13) 


\section{Revista}

CienciäoCuidado

Scientific Journal of Nursing

\section{Cuidados paliativos}

Desde otro punto de vista, los estudiantes mencionan que las intervenciones que emplearían con el paciente en etapa terminal hospitalizado y con los familiares serían técnicas y procedimientos para prevenir otras patologías y para el alivio del dolor, tales como: la administración de medicamentos $(02,04,09,14)$, mantener una buena dinámica de comunicación entre el paciente $\mathrm{y}$ la familia ${ }_{(01,06,10}$, siempre de forma respetuosa y con valores durante todo el proceso, para brindar un cuidado humano para una muerte digna ${ }_{(01,09,11,15,17)^{\circ}}$. Del mismo modo, hay que utilizar algunas técnicas espirituales religiosas ${ }_{(02,04)}$ y psicológicas para el manejo de emociones $_{(02,07,08,13,18)}$ con las cuales se podrá tener un mejor manejo del sufrimiento $(02,05,09)$ tanto del paciente como de los familiares. Los otros entrevistados establecen una diferencia entre el trato que le darían a un familiar en etapa terminal frente a un paciente con quien no tienen ningún vínculo afectivo ${ }_{(07)^{\circ}}$ No obstante, otros mencionan que de igual manera se debe realizar el cuidado de la mejor manera posible tratando de no involucrarse demasiado, para evitar que los afecte emocionalmente ${ }_{(15)}$.

"A lo mejor es muy diferente a que sea un paciente a que sea mi familiar y es tratar de consolar, de dar ánimo de confortar a la familia" (07).

"Darles todo el apoyo moral a sus familiares y a él, llevarlo a tener una muerte digna y que lleve a no mucho sufrimiento" ${ }_{(09)}$.

"No tanto involucrarme, porque me afectaría a mi, saber que es una persona que está en un estado terminal que sé que va a morir"

También, los entrevistados refieren que existe una diferencia entre el dolor y el sufrimiento; es decir, el dolor es una reacción física o fisiológica del cuerpo ante un estímulo $_{(02,04,05,08,14,15,17,18) \text {, }}$, refiriéndose a que es distinto al sufrimiento, que es más espiritual, psicoemocional y que relaciona el temor, la preocupación y el desgaste económico ${ }_{(02,04,05,08,09,14,17,18)}$ a un estado de agonía ${ }_{(10)}$. Otros indican que el dolor puede ser tanto físico como emocional $(01,10,13)$, pero que además uno depende del otro; de ahí, que el dolor prolongado produce sufrimiento y que ambos expresan un sentido de pérdida ${ }_{(03,06}$, $07,11,12,15)^{\circ}$

"No tan solo es físico, sino que también puede ser un dolor emocional o algo psicológico, que pueda provocarte, no sé, cambios en tu estado de ánimo y que tal
Ansiedad y nivel de conocimiento en cuidados palia-

tivos en estudiantes de Guerrero

vez muchos no lo perciban, pero lo está sintiendo esa persona" ${ }_{(01)}$.

"El dolor es más físico y el sufrimiento es más como emocional, podría ser más como una preocupación del paciente o se relaciona más con emociones como el miedo y el temor que pueda sentir ante la muerte, podría ser parte del sufrimiento o la preocupación de dejar a sus familiares podría ser parte del sufrimiento"

"El sufrimiento es el sentido de perecer, de perecer los sentimientos tristes o de algún malestar emocional" (07). "El dolor es algo que te produce sufrimiento, yo creo que el dolor y el sufrimiento es lo mismo" ${ }_{(11)}$.

Los cuidados paliativos son definidos por los estudiantes como técnicas e intervenciones en los pacientes terminales $(01,03,05,06,07,11,18)$ que se realizan para aliviar el dolor y el sufrimiento; algunos mencionan que los cuidados paliativos comprenden no solo la etapa terminal sino que se inicia desde los procesos de la enfermedad y estos son brindados por un equipo especializado ${ }_{(08)}$; por tanto en estos cuidados deben implementarse técnicas espirituales, psicoemocionales y clínicas $(04,13$, 17). A pesar de la importancia que tiene la familia durante este proceso, solo un entrevistado hace mención de la inclusión de la familia al momento de brindar el cuidado ${ }^{(15)}$.

"Son los cuidados que se les dan a las personas que se encuentran en una situación o problema de salud sin cura"

"Se implementan a un paciente que se encuentra con una enfermedad en etapa terminal y estos cuidados tienen el objetivo de mejorar la calidad de vida de ese paciente en ese momento" ${ }_{(02)}$.

"Cuidados proporcionados a las personas tanto como enfermas como en estado terminal, no precisamente tienen que estar en estado terminal para poder brindarles cuidados paliativos"

"Cuidados a las personas que están en el proceso de fallecimiento o de muerte brindarle apoyo emocional, psicológico, clínico en todas sus esferas que estuvieran afectadas" ${ }_{(13)}$.

En su mayoría los estudiantes refieren que el inicio de los cuidados paliativos es posterior al diagnóstico, es decir, cuando el paciente está en fase terminal $(02,03,04$, $05,06,07,08,09,10,11,13,14,15,16,17,18$; ; sin embargo, uno de los participantes indica que es a partir en que el paciente es dependiente y no puede realizar ninguna actividad independiente ${ }_{(01)}$. De su parte, otros estudiantes refieren 
que los cuidados culminan con la muerte del paciente $(03,04,05,06,07,08,09,10,11,14,15,16,17,18)$, a diferencia de quienes expresaron que terminarían posterior al proceso de duelo y terapia familiar ${ }_{(02,07,13)^{\circ}}$

"Empezarían desde el momento en que la persona está incapacitada para realizar el desarrollo de su vida normal y terminaría en el momento que ya está al borde de la muerte"

"Iniciarían desde el comienzo de la enfermedad y terminarían tanto en la recuperación o en la muerte del paciente y en cuestión al familiar, hasta que logre superar la pérdida"

"Empiezan desde el diagnóstico de la enfermedad que es en ese caso el cáncer y terminan en la fase final que es cuando el paciente ya fallece" (14).

Los hallazgos muestran que los estudiantes experimentan diferentes sentimientos y emociones al estar frente a las personas en estado terminal; igualmente mostraron seguridad y mencionan que es importante estar con el paciente y apoyarlo durante esa etapa ${ }_{12}$, darle fortaleza, mostrar empatía y responsabilidad, siempre se debe sonreír para mejorar su estado de ánimo $(01,03,16,18)^{\circ}$ A su vez, otros estudiantes refirieron sentir sufrimiento, dolor emocional ${ }_{(04,08,10)}$ y tristeza por la certeza de que el paciente fallecerá en algún momento $_{(02,05,06,07,09,11,14,15,17,18)}$; además expresaron que experimentan sentimientos de impotencia, compasión, desesperación, culpa y falta de esperanza por no poder ayudarlo $(02,03,06,10)^{\circ}$

"Compasión, empatía con los familiares, responsabilidad de que el paciente tenga los cuidados adecuados"

". "Nunca he estado con un paciente en ese estado terminal, pero me imagino no estar alegre, sino que estaría deprimida al estarlo viendo en la situación en la que se encuentra" ${ }_{(06)}$.

"Pues no podemos llorar en el servicio, a lo mejor y nos duele pero no precisamente demostrarlo frente a ellos" ${ }_{(07)}$.

"Quizás tenga un sentimiento de impotencia y sentiría un dolor emocional por la pérdida de una persona y por ver el dolor de los familiares" ${ }_{(10)}$.

"Cuando termina el sufrimiento "lo más bonito que puedes sentir es la ayuda que le diste en ese momento" (17)"

Algunos estudiantes mencionan que no están preparados para brindar cuidados paliativos y que no estan capacitados $_{(03,06,11,12)}$; otros mencionan que no se sienten capacitados completamente ${ }_{(17,18)}$; de manera similiar, algunos refieren tener el conocimiento teórico $\quad(09,10)$ pero que les hace falta la capacitación y la preparación

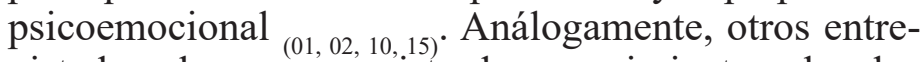
vistados alegan que existe desconocimiento sobre los cuidados, debido a que no cuentan con una unidad de aprendizaje en la que se les brinde capacitación ${ }_{(03,10)}$, además de que existe falta de experiencia para brindar estos cuidados

"Nunca nos toca en un hospital ver lo que otros vieron, siempre nos tocan pacientes diferentes y cuando deberíamos conocer algo tal vez no lo sabemos, porque no hemos tenido la experiencia o porque nunca hemos estudiado ese tipo de patología",

"La verdad no me siento capaz, pero puede ser que más adelante si lo pueda realizar o más bien tengo que poder brindar esos cuidados al paciente que lo necesite"

"Sí me siento preparado porque lo he vivido con familiares muy cercanos a mí, lo viví con mi abuela, le di cuidados paliativos, desgraciadamente falleció" ${ }_{(09)}$. "Para brindar cuidados paliativos al menos en conocimientos sí, pero emocionalmente quizás no, porque no he tenido la experiencia donde un paciente se muera o de ver el sufrimiento que tengan los familiares y pues quizás emocionalmente no" ${ }_{(10)}$.

"No sé cuáles son los cuidados paliativos, aquí se da esa optativa pero no en esta facultad, tienes que ir a psicología, asi que no me siento preparada para otorgarlos"

\section{Discusión}

La investigación en cuidados paliativos en estudiantes de enfermería permite inferir que no tienen un conocimiento acertado y profundo sobre esta temática, más cuando se entiende que serán la mayor fuerza de trabajo del sector salud, tanto público como privado.

Para la presente discusión se tomaron en cuenta los datos recabados por Siciliano \& Iglesias (23) quienes demostraron en su estudio que: el 54.1\% (n: 65) eran mujeres, $16.6 \%$ (n:20) habían realizado cursos previos, el 43.3\% (n:52) eran licenciados y el 56.6\% (n: 66) enfermeros. Los hallazgos de este estudio mostraron también que: el $20 \%$ de la población estudiada tuvo un conocimiento excelente, el $10 \%$ un conocimiento bueno y el resto tenía un conocimiento inadecuado. Además, se encontró un incremento insigni $\neg$ cante en la cantidad de respuestas correctas de los sujetos 


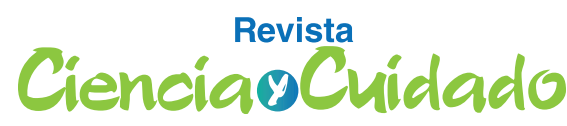

Scientific Journal of Nursing

que habían realizado cursos previos, comparados con quienes no los habían realizado ( $2.0 \pm 1.7$ vs. $2.7 \pm 2.4$; $\mathrm{p}=0.38$ ). Por otro lado, el doble de los encuestados que habían realizado cursos previos presentó un conocimiento adecuado en comparación con los que no tenían un conocimiento adecuado ( $30 \%$ vs $17.7 \%$; $=0.22)$; es decir, que comparado con el presente estudio en el que se encontró un $78.7 \%$ de conocimiento bajo, sin embargo, éste es mas alto.

Asimismo en un estudio realizado por Malagan et al., (24) en Lima, Perú, en estudiantes de la carrera de medicina, de la Universidad Peruana Cayetano Heredia, encontraron un nivel de conocimiento sobre cuidados paliativos en los alumnos de pre grado de sexto año adecuado, 114 de los participantes $(89.06 \%)$ calificado como bueno, a pesar de no contar con una materia afin a la medicina paliativa. Igualmente, en las unidades de aprendizaje que les enseñan tópicos relacionados con esta área, en comparación con los resultados encontrados en este estudio ( $78.7 \%$ conocimiento malo), es bajo, debido a que las unidades de aprendizaje en general no les proporcionan a los estudiantes las herramientas necesarias para hacer frente a este tipo de cuidado. Por otra parte, Guevara et al. (25), señalan que en las universidades en las que se forman profesionales de enfermería, aún no es visible la inclusión de programas o unidades de aprendizaje en las que de manera importante se consideren contenidos sobre Cuidados Paliativos, con el objetivo de capacitar a profesionales competentes para enfrentar los retos y solucionar las necesidades que demanda el paciente en fase terminal.

Hay que mencionar además, que la ansiedad ante la muerte de estudiantes de enfermería del presente estudio comparada con los resultados con estudiantes de medicina realizado por Boceta et al. (26), es de un $97,67 \%$ con puntuación moderada-alta (separado los puntajes en 5 niveles). De igual manera, en comparación con el $66.3 \%$ que son muy ansiosos encontrada en este estudio, los investigadores indicados más arriba hallaron una diferencia en el cotejo de los porcentajes de acuerdo a cada una de las dimisiones con respecto a: la propia muerte $21.77 \%$, proceso de morir $24.99 \%$, muerte de otros $27.15 \%$ y proceso de morir de otros $23.93 \%$, destacando una elevada ansiedad en el propio proceso de morir y la muerte de otros. También, al confrontar los resultados encontrados respecto a la experiencia sobre la propia muerte existe un mayor puntaje, aunque es moderadamente ansioso con un
Ansiedad y nivel de conocimiento en cuidados palia-

tivos en estudiantes de Guerrero
$55.3 \%$, para el propio proceso de morir 57.1\%, para el morir de otros $75.5 \%$ y para el proceso de morir de otros $60 \%$, denotando en este estudio que los puntajes más elevados se refieren a muy ansioso para la muerte de otros y el proceso de morir de otros.

Hay que mencionar, además que la investigación realizada por Costa et al. (27), muestra la importancia sobre la práctica de actividades en el aprendizaje de cuidados paliativos, la valorización de los equipos multidisciplinares, el sufrimiento involucrado en el proceso de autoidentificación con el paciente al final de la vida y el ciclo de empatía que resulta en la madurez emocional necesarios en cuidados paliativos; así mismo se destaca la importancia de formar profesionales capaces de manejar la subjetividad requerida para los cuidados paliativos.

De manera análoga, los hallazgos del estudio de Martínez et al. (28), muestran que la crisis de los cuidados o de los valores, incide en las percepciones de los estudiantes en aspectos tan relevantes como la responsabilidad frente al paciente para proveerle cuidados, olvidando la dimensión social de la enfermería.

Conforme el estudio de Rodríguez et al. (29), se derivan cinco categorías, que se centran en: la percepción, la interpretación, las creencias, los valores y la comprensión de los sentimientos durante la acción del cuidado humano. Comparado con los resultados del presente estudio en el que se incluyen dos categorías del análisis cualitativo que describen el nivel de conocimiento y ansiedad ante la muerte, se observa que los estudiantes experimentan sentimientos negativos ante la muerte y algunos refieren no estar preparados para brindar cuidados paliativos.

De acuerdo con el estudio de Hanzelíková Pogrányivá, quien descata que existe escasa preparación, afrontamiento ineficaz, miedos, valores y creencias; no obstante, señala que predomina la inquietud relacionada con el tratamiento del paciente y con la actitud obstinada del equipo de salud por salvar lo insalvable (30). Mientras que otros resultados muestran que las intervenciones oportunas de la enfermería en los cuidados paliativos, determinarán la calidad de vida de los pacientes (11).

\section{Conclusión}

La investigación realizada en escuelas de enfermería 
de la UAGro, muestra en los estudiantes, un nivel de conocimiento en cuidados paliativos malo y no se muestra diferencia significativa entre escuelas. Del mismo modo, el nivel de ansiedad ante la muerte tuvo una representación muy alta, puesto que los estudiantes se perciben muy ansiosos/as ante los procesos de muerte, siendo un mayor el nivel ante la muerte de otros y el proceso de morir de otros. Al relacionar las variables se encontró que existen dependencias muy significativas así como asociaciones y correlaciones de ambas variables. Además, se encontró que al relacionar estas últimas 2 variables con la del nivel de conocimiento, el grado de preocupación o ansiedad ante la muerte es mayor por la falta de conocimientos para afrontar los cuidados paliativos. De manera similar, hay que enfa- tizar que en general el plan educativo no le da al estudiante las herramientas necesarias para brindar este tipo de cuidado.

\section{Agradecimientos}

Gracias a las instituciones que dieron apertura para la realización de esta investigación y a los estudiantes que aceptaron participar en el estudio.

\section{Conflicto de Intereses}

Los autores declaran no tener ningún conflicto de intereses

\section{Referencias Bibliograficas}

1. SSA. Programa nacional de capacitación en cuidados paliativos para equipos de primer contacto en atención primaria. Abril de 2016, en la Ciudad de México. http://www.calidad.salud.gob.mx/site/calidad/docs/programa cuidados paliativos.pdf

2. Wenk R, De Lima L, Mutto E, Berenguel MdR, Centeno C. Encuestro sobre educación de cuidados paliativos en Latinoamérica. Recomendaciones sobre enseñanza. Medicina Paliativa. 2016; 23(1):42-48. http://dx.doi. org/10.1016/j.medipa.2013.11.002

3. Real Academia Española. Diccionario de la Real Academia Española. [Online].; 2018 [cited 2019 Marzo 20. Available from: https://dle.rae.es/srv/fetch?id=AMrJ4zs

4. Esparza-Parga R, Rubio-Barrios J. La pregunta por el conocimiento. Revista Multidisciplinaria del Consejo de Investigación de la Universidad de Oriente. 2016; 28(4):813-818. http://www.redalyc.org/articulo. oa? id $=427751143015$

5. Organización Mundial de la Salud. OMS. [Online]; 2017 [cited 2019 Marzo 20. Available from: https://www. who.int/cancer/palliative/es/

6. Pueyo-Garrigues S, Pueyo-Garrigues M, Pardavila-Belio MI. Necesidades de los familiares de un paciente terminal institucionalizado en un centro geriátrico: caso clínico. Gerokomos [Internet]. 2015 Sep [citado 2021 Dic 17]; 26(3):94-96. Disponible en: https://dx.doi.org/10.4321/S1134-928X2015000300005

7. Red Estatal de Salud Mental. Revista del Instituto Jalisciense de Salud Mental. Dossier. Trastornos de ansiedad. Salme número 12 / enero-junio de 2019. https://salme.jalisco.gob.mx/sites/salme.jalisco.gob.mx/files/ salme 12.pdf

8. Freitas-Tiago LL, Banazeski AC, Eisele A, de Souza EN, Bitencourt JV, Souza SS. La visión de enfermería del proceso de muerte y muerte del paciente crítico: una revisión integradora. Enfermo. glob. [Internet]. Enero de 2016 [consultado el 17 de diciembre de 2021]; 15 (41):322-334. Disponible en: http://scielo.isciii.es/scielo. php?script $=$ sci_arttext\&pid $=$ S1695-61412016000100015\&lng=es

9. Organización Mundial de la Salud. OMS. [Online].; 2017 [cited 2018 Diciembre 1. Available from: https:// www.who.int/features/factfiles/palliative-care/es/

10. Organización Mundial de la Salud. Cuidados paliativos. Datos y cifras. 20 de agosto de 2020. https://www. who.int/es/news-room/fact-sheets/detail/palliative-care 
11. Viaggio C, Etcheverry LP. Enfermería en cuidados paliativos. Humanizar el final de la vida, Red Sociales, Revista del Departamento de Ciencias Sociales. 2021; 8(2):10-20. http:/www.redsocialesunlu.net/wp-content/ uploads/2021/05/3.-Enfermer\%C3\%ADa-en-cuidados-paliativos.pdf

12. Fhon JRS, Silva LM, Rodrigues RAP, Carhuapoma AME. Percepción de las enfermeras sobre cuidados paliativos: experiencia con pacientes oncológicos. Rev. iberoam. Educ. investi. Enferm. 2018; 8(3):28-36.

13. Guerrero-Castañeda RF, Chávez-Urías RA. Momento de cuidado, un encuentro fenomenológico entre enfermera y persona cuidada: reflexión en Watson. Cultura de los Cuidados (Edición digital), 24 (58). Recuperado de http://dx.doi.org/10.14198/cuid.2020.58.02

14. Del Valle-Fayos L, Palop-Muñoz J. El cuidado invisible. Necesidades psicoemocionales del paciente critico en Unidades de Cuidados Intensivos. Etica de los Cuidados. [Internet] 2018. [acceso: 17/12/2021] Disponible en: http://ciberindex.com/c/et/e11388

15. Ramírez P, Müggenburg C. Relaciones personales entre la enfermera y el paciente. Enfermo. univ [revista de Internet]. Septiembre de 2015 [consultado el 17 de diciembre de 2021]; 12(3):134-143. Disponible en: https:// doi.org/10.1016/j.reu.2015.07.004

16. Garza N, Rodríguez MT. Las palabras de los otros. Fragmentos sobre la muerte. Universidad Nacional Autónoma de México, Mexico. Andamios, 2017; 14(33):151-184. [fecha de Consulta 17 de Diciembre de 2021]. Disponible en: https://www.redalyc.org/articulo.oa?id=62849641008

17. Álvarez SS, Vargas MAO, Zilli F. Fortalezas y debilidades para afrontar el proceso de morir y la muerte: reflexiones de los estudiantes. Texto Contexto Enferm [Internet]. 2020; 29(Spe):e20190257. Disponíble en: https://doi.org/10.1590/1980-265X-TCE-2019-0257

18. Vega-Ayasta MT, Díaz-Manchay RJ, Cervera-Vallejos MF, Rodríguez-Cruz LD, Tejada-Muñoz S, Guerrero-Quiroz SE. Amabilidad, confort y espiritualidad en los cuidados paliativos oncológicos: Aporte para la humanización en salud. Cultura de los Cuidados (Edición digital), 2020; 24(58). Recuperado de: http://dx.doi. org/10.14198/cuid.2020.58.05

19. Soikkeli-Jalonen A, Stolt M, Hupli M, Lemetti T, Kennedy C, Kydd A, Haavisto E. Instruments for assessing nurses' palliative care knowledge and skills in specialised care setting: an integrative review. Journal of clinical nursing [online], 29(5-6):736-757. Available from: https://doi.org/10.1111/jocn.15146

20. Mondragon-Sanchez EJ, Landeros-Olvera E, Pérez-Noriega E. Validación de la Escala de Miedo a la Muerte de Collett - Lester en estudiantes universitarios de enfermería de México. MedUNAB [Internet]. 31 de marzo de 2020 [citado 17 de diciembre de 2021];23(1):11-2. Disponible en: https://revistas.unab.edu.co/index.php/ medunab/article/view/3723

21. Colanzi I. Testimonios: nuevos desafíos de la metodología cualitativa en investigación. Informe de Congreso. La Plata, Argentina: Universidad Nacional de la Plata, Facultad de Psicologia, Centro interdiciplinario de Investigación en Genero; 2015. http://sedici.unlp.edu.ar/handle/10915/56317

22. Cámara de diputados del H. Congreso de la Unión. Ley General de Salud. Diario Oficial de la Federación el 7 de febrero de 1984. TEXTO VIGENTE. Última reforma publicada DOF 07-01-2021. https://www.diputados. gob.mx/LeyesBiblio/pdf_mov/Ley_General_de_Salud.pdf

23. Siciliano CA, Iglesias A. Estudio transversal para definir el grado de conocimiento en cuidados paliativos del servicio de enfermería en una clínica privada. Fronteras en Medicina 2020; 15(3):159-164. https://DOI. org/10.31954/RFEM/202003/0159-0164

24. Málagan-Rodriguez G, Ordoñez-Molero DA, Rivera-Muñoz AE, Mateluna-Paredes PC. Nivel de conocimiento acerca de cuidados paliativos en alumnos de medicina de sexto año de la Universidad Peruana Caye- 
Scientific Journal of Nursing

tano Heredia. 2018. https://hdl.handle.net/20.500.12866/1505

25. Guevara-Valtier MC, Santos-Flores JM, Santos-Flores I, Valdez-Ramírez FJ, Garza-Dimas IY, Paz-Morales MÁ, et al. Conocimiento de enfermería sobre cuidados paliativos en centros de primer y segundo nivel de atención para la salud. Revista CONAMED. 2017 Noviembre; 22(4):170-173. https://www.medigraphic.com/ pdfs/conamed/con-2017/con174d.pdf

26. Boceta-Osuna J, Galán-Gonzáles-Serna JM, Gamboa-Antiñolo FM, Muniain-Ezcurra MA. Factores sociodemograficos que influyen en la ansiedad ante la muerte en estudiantes de medicina. Educación Médica. 2017; 18(3):179-187. https://doi.org/10.1016/j.edumed.2016.07.004

27. Costa P, Poles K, Silva AE. Formación en cuidados paliativos: La experiencia de estudiantes de Medicina y Enfermería. INTERFACE. 2016 Mayo 3; 20(59):1041-1052. https://doi.org/10.1590/1807-57622015.0774

28. Martinez-Rodríguez L, Venceslao-Pueyo M, Ramió I, Jofre A. Crisis de cuidado: percepción del cuidado en los estudiantes de enfermería. Psicoperspectivas. 2016; 15(3):135-145. https://dx.doi.org/10.5027/psicoperspectivas-Vol15-Issue3-fulltext-777

29. Rodríguez L, Rondón R, Varón M, Guerra A, Fernández V, Lorenzini Erdmann A. Enfoque fenomenológico del cuidado humano en estudiantes de enfermería. SALUS. 2015 Mayo-Agosto; 19(2):7-12. Disponible en: http://ve.scielo.org/scielo.php?script=sci_arttext\&pid=S1316-71382015000200003\&lng=es

30. Marrero-González CM, García-Hernández AM. Vivencias de las enfermeras ante la muerte. Una revisión. Ene. [Internet]. 2019 [citado 2021 Dic 17] ; 13(2):1321. Disponible en: http://scielo.isciii.es/scielo. php?script $=$ sci arttext\&pid=S1988-348X2019000200001\&lng=es 